TRANSACTIONS OF THE

AMERICAN MATHEMATICAL SOCIETY

Volume 173, November 1972

\title{
ON A VARIATION OF THE RAMSEY NUMBER
}

\author{
BY \\ GARY CHARTRAND $\left.{ }^{1}\right)$ AND SEYMOUR SCHUSTER $\left({ }^{2}\right)$
}

ABSTRACT. Let $c(m, n)$ be the least integer $p$ such that, for any graph $G$ of order $p$, either $G$ has an $m$-cycle or its complement $\bar{G}$ has an $n$-cycle. Values of $c(m, n)$ are established for $m, n \leq 6$ and general formulas are proved for $c(3, n), c(4, n)$, and $c(5, n)$.

Introduction. It is a well-known fact that in any gathering of six people, there are three people who are mutual acquaintances or three people who are mutual strangers. This statement has the graph-theoretic formulation that either a given graph of order 6 or its complement contains a triangle. It might further be mentioned that " 6 " is minimum with respect to this property.

The Ramsey number $r(m, n)$ may be considered a generalization of the above observation. For integers $m, n \geq 2$, the number $r(m, n)$ is defined as the smallest positive integer $p$ such that given any graph $G$ of order $p$, either $G$ contains the complete subgraph $K_{m}$ of order $m$ or the complement $\bar{G}$ of $G$ contains $K_{n}$. Hence, the aforementioned fact states that $r(3,3)=6$. One may easily note that $r(m, n)=$ $r(n, m)$ and that $r(2, n)=n$ for all $n \geq 2$.

It is a result due to Ramsey [3] that the number $r(m, n)$ exists for all $m, n \geq 2$. Despite the fact that a great deal of research has been done on Ramsey numbers, only six values $r(m, n)$ have been determined for $m, n \geq 3$ (see [1]); namely, $r(m, n)$ is known (for $m, n \geq 3)$ only when $(m, n)=(3,3),(3,4),(3,5),(3,6)$, $(3,7),(4,4)$.

If we denote an $n$-cycle (a cycle of length $n$ ) by $C_{n}$, the original problem may be stated as: Given a graph $G$ of order 6 , either $G$ or $\bar{G}$ contains a 3-cycle (triangle). This suggests a generalization different from that which leads to the Ramsey numbers. For $m, n \geq 3$, we define the number $c(m, n)$ to be the least positive integer $p$, such that for any graph $G$ of order $p$, either $G$ contains the m-cycle $C_{m}$ or $\bar{G}$ contains $C_{n}$. Of course, we have $c(3,3)=6$. The number $c(m, n)$ always exists since $c(m, n) \leq r(m, n)$. It is the object of this paper to determine the value of $c(m, n)$ for several pairs $(m, n)$; in particular, $c(3, n), c(4, n)$, and $c(5, n)$ are determined for all $n \geq 3$. Before proceeding further, we present a few definitions and some additional notation. All terms not defined here may be found in [2].

Received by the editors May 17, 1971.

AMS (MOS) subject classifications (1970). Primary 05C 35.

Key words and phrases. Graph, cycle, Ramsey number, complement.

$\left({ }^{1}\right)$ Research supported in part by the Office of Naval Research.

$\left({ }^{2}\right)$ Research supported by the National Science Foundation. 
The complete bipartite graph $K(m, n), m, n \geq 1$, is that graph $G$ of order $m+n$, whose vertex set may be partitioned as $V_{1} \cup V_{2}$ such that $\left|V_{1}\right|=m,\left|V_{2}\right|=n$ and $e=u v$ is an edge of $G$ if and only if $u \in V_{i}$ and $v \in V_{j}, i \neq j$. For connected graphs $G_{1}$ and $G_{2}$, we define $G_{1} \cup G_{2}$ to be the disconnected graph having the two components $G_{1}$ and $G_{2}$. Note that if $G=K(m, n)$, then $\bar{G}=K_{m} \cup K_{n}$.

The numbers $c(3, n)$. We have already mentioned that $c(3,3)$ is the wellknown Ramsey number $r(3,3)=6$. We consider $c(3,4)$ next.

Theorem 1. $c(3,4)=7$.

Proof. Let $H=K(3,3)$ so that $\bar{H}=K_{3} \cup K_{3}$. The graph $H$ contains no 3-cycle and its complement $\bar{H}$ fails to contain a 4-cycle; thus, $c(3,4) \geq 7$. To verify that $c(3,4)=7$, we let $G$ be an arbitrary graph of order 7 and assume $G$ contains no 3cycle. We show that $\bar{G}$ contains a 4 -cycle.

Since $c(3,3)=6$, either $G$ or $\bar{G}$ has a 3 -cycle; hence, $\bar{G}$ contains a 3 -cycle, which we represent as $C: u_{1}, u_{2}, u_{3}, u_{1}$. (See Figure 1a, where the edges of $\bar{G}$ are represented by dashed lines.) Denote the remaining vertices by $v_{1}, v_{2}, v_{3}$, and $v_{4}$. If some $v_{i}$ is joined in $\bar{G}$ to more than one vertex of $C$, then $\bar{G}$ contains a 4-cycle. We may assume, then, that each $v_{i}$ is adjacent in $G$ to at least two vertices of $C$. This implies that every two distinct $v_{i}$ must be joined in $G$ to a common vertex of $C$. (See Figure $1 b$, where the edges of $G$ are represented by solid lines.) Because $G$ contains no triangles, every two distinct $v_{i}$ must be adjacent in $\bar{G}$ (see Figure 1c) which implies that $\bar{G}$ contains $K_{4}$ and hence $C_{4}$ as a subgraph.

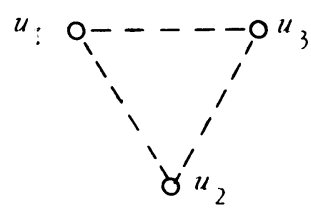

a

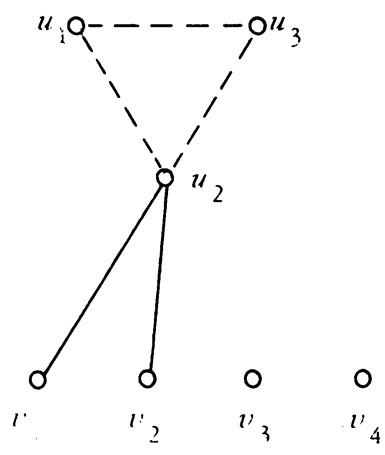

b

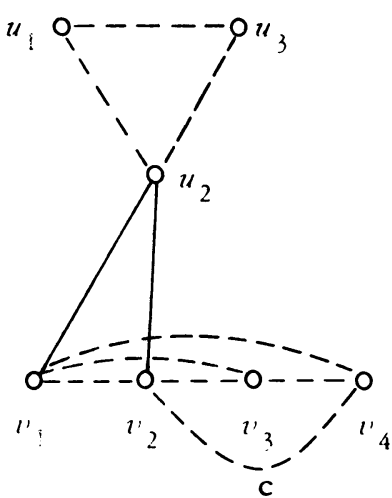

Figure 1

We now proceed to the general situation.

Theorem 2. For $n \geq 4, c(3, n)=2 n-1$.

Proof. First, we note that if $H=K(n-1, n-1)$ so that $\bar{H}=K_{n-1} \cup K_{n-1}$, then $H$ contains no $3-c y c l e s$ and $\bar{H}$ contains no $n$-cycles. Thus, $c(3, n) \geq 2 n-1$. We prove that $c(3, n)=2 n-1$, for all $n \geq 4$, by using induction on $n$. 
By Theorem $1, c(3,4)=7$. Assume that $c(3, n)=2 n-1$ for some $n \geq 4$. It follows, therefore, that if $F$ is any graph of order $2 n-1$, either $F$ contains a 3cycle or $\bar{F}$ contains an $n$-cycle. We now show that $c(3, n+1)=2 n+1$. Let $G$ be a graph of order $2 n+1$, and assume $G$ has no 3 -cycles. Because $c(3, n+1) \geq$ $2 n+1$, it suffices to prove that $\bar{G}$ contains an $(n+1)$-cycle. Since, by the induction hypothesis, $c(3, n)=2 n-1$, the graph $\bar{G}$ contains an $n$-cycle, say $C: u_{1}, u_{2}$, $\cdots, u_{n}, u_{1}$. Designate the remaining vertices by $v_{1}, v_{2}, \cdots, v_{n}$, and $v_{n+1}$.

Suppose that some vertex $u_{i}, 1 \leq i \leq n$, is adjacent in $G$ to all vertices $v_{j}$, $j=1,2, \cdots, n+1$. Since $G$ contains no 3 -cycles, every two distinct vertices $v_{j}$ are adjacent in $\bar{G}$. However, this implies that $\bar{G}$ contains $K_{n+1}$ and therefore $C_{n+1}$ as a subgraph. We henceforth assume that for $i=1,2, \cdots, n$, the vertex $u_{i}$ is adjacent in $\bar{G}$ to some $v_{j}$.

We now consider two cases.

Case 1. Suppose there exist two alternate vertices on $C$ which are respective. $l_{y}$ joined in $\bar{G}$ to two distinct $v_{i}$. Assume $u_{1} v_{1}, u_{3} v_{3} \in E(\bar{G})$. If some vertex $v_{i}$ is joined in $\bar{G}$ to two consecutive vertices of $C$, then $\bar{G}$ contains an $(n+1)$-cycle. Otherwise we have $u_{2} v_{1}, u_{2} v_{3} \in E(G)$, which implies that $v_{1} v_{3} \in E(\bar{G})$. However, then, $\bar{G}$ contains the $(n+1)$-cycle $u_{1}, v_{1}, v_{3}, u_{3}, u_{4}, \cdots, u_{n}, u_{1}$.

Case 2. Suppose no two alternate vertices on $C$ are joined in $\bar{G}$ to distinct vertices $v_{i}$. This implies that $u_{1}$ and $u_{3}$ are joined in $\bar{G}$ to the same $v_{i}$, say $v_{1}$. Indeed, every $u_{i}$, with $i$ odd, is joined in $\bar{G}$ to $v_{1}$. If $n$ is odd, then $v_{1}$ is joined in $G$ to both $u_{1}$ and $u_{n}$ which produces an $(n+1)$-cycle in $\bar{G}$. Assume that $n$ is even. It follows here that each $u_{i}$, with $i$ even, is adjacent in $\bar{G}$ to the same $v_{i} \neq$ $v_{1}$, say $v_{2}$.

Each $v_{j}, 3 \leq j \leq n+1$, is necessarily joined in $G$ to every vertex of $C$; otherwise, we revert back to Case 1 . Since $G$ contains no triangles, $v_{i}$ and $v_{j}, 3 \leq i<$ $j \leq n+1$, are adjacent in $\bar{G}$. For the same reason and because $v_{1} u_{2}, v_{2} u_{1} \in E(G)$, all edges $v_{1} v_{i}$ and $v_{2} v_{i}, 3 \leq i \leq n+1$, belong to $\bar{G}$. Now $v_{1}, v_{3}, v_{2}, v_{4}, v_{5}, \cdots$, $v_{n+1}, v_{1}$ is a desired $(n+1)$-cycle in $\bar{G}$.

The numbers $c(4, n)$. As $c(m, n)=c(n, m)$, it follows that $c(4,3)=7$ by Theorem 1. Thus we need only consider $c(4, n)$ for $n \geq 4$. Since the values of $c(4,4)$ and $c(4,5)$ do not follow the general formula which we present in this section, we must establish these numbers individually. We begin by doing this.

Theorem 3. $c(4,4)=6$.

Proof. Let $H=C_{5}$ so that $\bar{H}=C_{5}$. Since neither $H$ nor $\bar{H}$ contains a 4-cycle, $c(4,4) \geq 6$. Let $G$ be a graph of order 6 , and assume neither $G$ nor $\bar{G}$ contains a 4-cycle. Because $=(3,3)=6$, either $G$ or $\bar{G}$ contains a triangle. Without loss of generality, we assume $G$ contains the 3-cycle $C: u_{1}, u_{2}, u_{3}, u_{1}$. Denote the other vertices of $G$ by $v_{1}, v_{2}$, and $v_{3}$. No vertex $v_{i}$ can be joined in $G$ to more than one 
vertex of $C$, for otherwise $G$ contains a 4-cycle. Hence each $v_{i}$ is adjacent in $\bar{G}$ to at least two vertices of $C$. If there exist two $v_{i}$ which are adjacent in $\bar{G}$ to the same two vertices of $C$, then $\bar{G}$ contains a 4-cycle. Hence, we suppose $v_{1}$ is ad. jacent in $\bar{G}$ to $u_{1}$ and $u_{2}, v_{2}$ is adjacent in $\bar{G}$ to $u_{2}$ and $u_{3}$, and $v_{3}$ is adjacent in $\bar{G}$ to $u_{1}$ and $u_{3}$; moreover, $v_{1} u_{3}, v_{2} u_{1}, v_{3} u_{2} \in E(G)$. No two $v_{i}$ are adjacent in $G$, for then $G$ has a 4-cycle. This implies that $v_{1} v_{2}, v_{1} v_{3}, v_{2} v_{3} \in E(\bar{G})$, but then $v_{1}$, $u_{2}, v_{2}, v_{3}, v_{1}$ is a $4 \cdot$ cycle in $\bar{G}$ which produces a contradiction.

Theorem 4. $c(4,5)=7$.

Proof. Let $H=K_{3} \cup K_{3}$ so that $\bar{H}=K(3,3)$. The graph $H$ has no 4-cycle and $\bar{H}$ has no 5 -cycle; thus, $c(4,5) \geq 7$. Let $G$ be a graph of order 7 , and assume $G$ has no 4-cycle. We prove that $\bar{G}$ contains a 5 -cycle.

Since $c(4,4)=6$ by Theorem 3 , the graph $\bar{G}$ contains a 4-cycle, say $C: u_{1}$, $u_{2}, u_{3}, u_{4}, u_{1}$. Let $v_{1}, v_{2}$, and $v_{3}$ denote the other vertices. If any of $v_{1}, v_{2}$, and $v_{3}$ is adjacent in $\bar{G}$ to two consecutive vertices of $C$, then $\bar{G}$ contains a 5. cycle. Suppose, then, that none of $v_{1}, v_{2}$, and $v_{3}$ is adjacent in $\bar{G}$ to consecutive vertices of $C$. Hence each $v_{i}$ is joined in $G$ to opposite vertices of $C$. Necessarily, there exist two $v_{i}$, say $v_{1}$ and $v_{2}$, which are adjacent in $G$ to the same opposite vertices of $C$, say $u_{1}$ and $u_{3}$. The graph $G$ therefore contains the 4-cycle $u_{1}$, $v_{1}, u_{3}, v_{2}, u_{1}$, which is contrary to hypothesis.

In order to determine a general formula for $c(4, n)$, we establish the number $c(4,6)$.

Theorem 5. $c(4,6)=7$.

Proof. Let $H=K(1,5)$; thus, $\bar{H}=K_{1} \cup K_{5}$. Because $H$ has no cycles (hence no $4-c y c l e s)$ and $\bar{H}$ has no 6-cycles, $c(4,6) \geq 7$. Let $G$ be a graph of order 7 , and assume $G$ has no 4 -cycles. We show that $\bar{G}$ contains a 6 -cycle. Since $c(4,5)=7$ by Theorem 4 and since $G$ has no 4 -cycles, it follows that $\bar{G}$ has a 5-cycle $C: u_{1}$, $u_{2}, u_{3}, u_{4}, u_{5}, u_{1}$. Let the remaining vertices be denoted by $v_{1}$ and $v_{2}$.

If $v_{1}$ or $v_{2}$ is adjacent in $\bar{G}$ to two consecutive vertices of $C$, then $\bar{G}$ has a 6acycle. Assume neither $v_{1}$ nor $v_{2}$ is adjacent in $\bar{G}$ to consecutive vertices of $C$ so that each of $v_{1}$ and $v_{2}$ is joined in $G$ to a set of three vertices of $C$ (not all consecutive). If there exist two vertices of $C$ joined in $G$ to both $v_{1}$ and $v_{2}$, then $G$ has a $4-c y c l e$ which produces a contradiction. However, there must exist one vertex of $C$ joined in $G$ to $v_{1}$ and $v_{2}$; hence we assume, without loss of generality, that $v_{1}$ and $v_{2}$ are joined in $G$ to $u_{1}$, the edges $v_{1} u_{2}, v_{1} u_{4}, v_{2} u_{3}, v_{2} u_{5} \in E(G)$, while $v_{1} u_{3}, v_{1} u_{5}, v_{2} u_{2}, v_{2} u_{4} \in E(\bar{G})$. Now $\bar{G}$ contains the 6 -cycle $v_{1}, u_{3}, u_{2}, v_{2}$, $u_{4}, u_{5}, v_{1}$.

We are now prepared to determine the remaining values of $c(4, n)$.

Theorem 6. For $n \geq 6, c(4, n)=n+1$. 
Proof. Let $n \geq 6$ and let $H=K(1, n-1)$ so that $\bar{H}=K_{1} \cup K_{n-1}$. The graph $H$ has no 4 -cycles and its complement $\bar{H}$ has no $n$-cycles; therefore, $c(4, n) \geq n+1$. We proceed by induction on $n(\geq 6)$. That $c(4,6)=7$ is the result of Theorem 5 . Assume that, for some $n \geq 6, c(4, n)=n+1$; hence, for every graph $F$ of order $n+1$, either $F$ contains a $4-c y c l e$ or $\bar{F}$ contains an $n$-cycle. We consider the number $c(4, n+1)$. Since $c(4, n+1) \geq n+2$, it suffices to prove that if $G$ is a graph of order $n+2$, either $G$ has a $4-$ cycle or $\bar{G}$ has an $(n+1)$-cycle. Suppose $G$ does not contain a 4 -cycle. Since $c(4, n)=n+1$ by the induction hypothesis, it follows that $\bar{G}$ contains an $n$-cycle, say $C: u_{1}, u_{2}, \cdots, u_{n}, u_{1}$. Designate the other two vertices by $v_{1}$ and $v_{2}$.

If $v_{1}$ or $v_{2}$ is adjacent in $\bar{G}$ to consecutive vertices of $C$, then $\bar{G}$ contains an $(n+1)$-cycle, completing the proof. Assume, therefore, that neither $v_{1}$ nor $v_{2}$ is adjacent in $\bar{G}$ to consecutive vertices of $C$, which implies that each of $v_{1}$ and $v_{2}$ is adjacent in $G$ to some set of $\{n / 2\}$ vertices of $C$ such that the set contains at least one of every two consecutive vertices of $C$. If $v_{1}$ and $v_{2}$ are adjacent in $G$ to the same two (or more) vertices of $C$, then $G$ contains a $4-c y c l e$, which is contradictory. Thus we assume that $v_{1}$ and $v_{2}$ are mutually adjacent in $G$ to one or no vertices of $C$. We consider the se two cases.

Case 1. Assume that $v_{1}$ and $v_{2}$ are mutually adjacent in $G$ to no vertices of $C$. In this case, it necessarily follows that each of $v_{1}$ and $v_{2}$ is joined in $G$ to exactly $n / 2$ vertices of $C$ such that neither $v_{1}$ nor $v_{2}$ is adjacent in $G$ to two consecutive vertices of $C$. Hence, $n$ is even and, without loss of generality, we as. sume $v_{1}$ is joined in $G$ to the vertices of $S_{1}=\left\{u_{i} \mid i\right.$ is odd $\}$ and $v_{2}$ is joined in $G$ to the vertices of $S_{2}=\left\{u_{i} \mid i\right.$ is even $\}$. Therefore, $v_{1}$ is joined in $\bar{G}$ to the elements of $S_{2}$, and $v_{2}$ is adjacent in $\bar{G}$ to the elements of $S_{1}$. If all edges $u_{i} u_{j}$, with $i$ and $j$ odd, belong to $G$, then since $n \geq 6, G$ contains the 4 -cycle $u_{1}, v_{1}$, $u_{3}, u_{5}, u_{1}$, which is contrary to hypothesis. Therefore, $\bar{G}$ contains an edge $u_{i} u_{j}$ with $i$ and $j$ odd such that $1 \leq i<j<n$, say. The graph $\bar{G}$ thus contains the $(n+1)$-cycle $u_{i}, u_{j}, u_{j-1}, \cdots, u_{i+1}, v_{1}, u_{j+1}, u_{j+2}, \cdots, u_{i-1}, u_{i}$.

Case 2. Assume that $v_{1}$ and $v_{2}$ are mutually adjacent in $G$ to exactly one vertex of $C$, say $u_{1}$. Exactly one of $v_{1}$ and $v_{2}$ is adjacent in $G$ to $u_{2}$, for if this were not the case, then $v_{1}$ and $v_{2}$ must be joined in $G$ to $u_{3}$, which is contrary to our assumption. Without loss of generality, we suppose that $v_{1} u_{2} \in E(G)$. Neco essarily, $v_{2} u_{3} \in E(G)$ or else $v_{2}$ is joined in $\bar{G}$ to the consecutive vertices $u_{2}$ and $u_{3}$ of $C$, which we have previously ruled out. By the same reasoning, $v_{1} u_{4} \in E(G)$, $v_{2} u_{5} \in E(G)$, etc. Hence, if we let $S_{1}$ and $S_{2}$ be defined as in Case 1 , then $v_{1}$ is joined in $G$ to the elements of $\left\{u_{1}\right\} \cup S_{2}$ and joined in $\bar{G}$ to the vertices of $S_{1}$ $\left\{u_{1}\right\}$, while $v_{2}$ is joined in $G$ to the vertices of $S_{1}$ and joined in $\bar{G}$ to the vertices of $S_{2}$.

If $G$ contains all edges $u_{i} u_{j}$ with $i$ and $j$ even, then since $n \geq 6, G$ contains 
the 4-cycle $v_{1}, u_{2}, u_{4}, u_{6}, v_{1}$ which produces a contradiction. Therefore, $\bar{G}$ contains some edge $u_{i} u_{j}$, where $i$ and $j$ are even and $1<i<j \leq n$, say. The graph $G$ then has the $(n+1)$-cycle $u_{j}, u_{i}, u_{i+1}, \cdots, u_{j-1}, v_{1}, u_{i-1}, u_{i-2}, \cdots, u_{j+1}, u_{j}$, which yields the desired result.

The numbers $c(5, n)$. We have already established the value of $c(5, n)$ for $n=3$ and $n=4$. In order to present a formula for $c(5, n), n \geq 5$, we shall first de. termine $c(5,5)$.

Theorem 7. $c(5,5)=9$.

Proof. Let $H=K(4,4)$ so that $\bar{H}=K_{4} \cup K_{4}$. Neither $H$ nor $\bar{H}$ contains a 5cycle; thus, $c(5,5) \geq 9$. Let $G$ be a graph of order 9 , and assume that neither $G$ nor $\bar{G}$ has a 5 -cycle.

Since $c(4,4)=6$, at least one of $G$ and $\bar{G}$ contains a 4 -cycle. Without loss of generality, we as sume that $G$ contains the 4 -cycle $C: u_{1}, u_{2}, u_{3}, u_{4}, u_{1}$. Denote the other vertices of $G$ by $v_{1}, v_{2}, v_{3}, v_{4}$, and $v_{5}$. No $v_{i}$ is adjacent in $G$ to two consecutive vertices of $C$ since $G$ contains no 5-cycle. Hence each $v_{i}$ is joined in $\bar{G}$ to two opposite vertices of $C$.

Since $G$ does not contain a 5-cycle, $G$ does not contain $K_{5}$ as a subgraph; therefore, not every two distinct $v_{i}$ are adjacent in $G$. Assume, say, that $v_{1} v_{2} \epsilon$ $E(\bar{G})$. If there are two vertices $u_{i}$ and $u_{j}$ with $v_{1} u_{i}, v_{2} u_{j} \in E(\bar{G})$ such that there exists a vertex $v_{k}, 3 \leq k \leq 5$, with $v_{k} u_{i}, v_{k} u_{j} \in E(\bar{G})$, then $\bar{G}$ contains a 5-cycle, which produces a contradiction. Hence, no such vertices $u_{i}, u_{j}$, and $v_{k}$ exist in $\bar{G}$. We now consider two cases, assuming throughout that $v_{1} v_{2} \in E(\bar{G})$.

Case 1. Assume $v_{1}$ and $v_{2}$ are adjacent in $\bar{G}$ to the same pair of opposite vertices of $C$, say $u_{1}$ and $u_{3}$. None of $v_{3}, v_{4}$, and $v_{5}$ is joined in $\bar{G}$ to both $u_{1}$ and $u_{3}$, for then we have conditions which produce a 5 -cycle, as described above. Therefore, each of $v_{3}, v_{4}$, and $v_{5}$ is joined in $\bar{G}$ to $u_{2}$ and $u_{4}$. Now $v_{3} v_{4} \in E(G)$, for otherwise $v_{3}, v_{4}, u_{2}, v_{5}, u_{4}, v_{3}$ is a 5-cycle in $\bar{G}$. Similarly, $v_{3} v_{5}, v_{4} v_{5} \epsilon$ $E(G)$.

If $v_{1}$ is joined in $\bar{G}$ to one of $v_{3}, v_{4}$, and $v_{5}$, and $v_{2}$ is joined in $\bar{G}$ to some other vertex of $v_{3}, v_{4}$, and $v_{5}$, then $\bar{G}$ contains a 5-cycle. Since $\bar{G}$ has no 5cycles, it follows that one of $v_{1}$ and $v_{2}$ is joined in $G$ to all of $v_{3}, v_{4}$, and $v_{5}$, say $v_{2} v_{3}, v_{2} v_{4}, v_{2} v_{5} \in E(G)$. If there are two edges of $G$ from the vertices of $C$ to two distinct vertices of $v_{3}, v_{4}$, and $v_{5}$, then $G$ contains a 5-cycle. Because $G$ cannot contain a 5 -cycle, there must exist a vertex among $v_{3}, v_{4}$, and $v_{5}$, say $v_{5}$, which is joined in $\bar{G}$ to all vertices of $C$. Thus, $\bar{G}$ contains the 5 -cycle $v_{5}, u_{3}$, $v_{1}, v_{2}, u_{1}, v_{5}$, which produces a contradiction.

Case 2. Assume $v_{1}$ is adjacent in $\bar{G}$ to $u_{1}$ and $u_{3}$, and $v_{2}$ is adjacent in $\bar{G}$ to $u_{2}$ and $u_{4}$. Suppose $v_{3}$ is adjacent in $\bar{G}$ to $u_{1}$ and $u_{3}$. Necessarily, $v_{3}$ is adjacent in $G$ to $u_{2}$ and $u_{4}$, for otherwise we have conditions sufficient to produce a 
5-cycle in $\bar{G}$, as mentioned earlier. For the same reason, $v_{2} u_{1}, v_{2} u_{3} \in E(G)$. The vertex $v_{1}$ is joined in $G$ to $u_{2}$ or $u_{4}$, for otherwise we return to Case 1 . Thus, we assume $v_{1} u_{2} \in E(G)$. Now $v_{1} v_{3} \in E(\bar{G})$, or else $v_{1}, v_{3}, u_{4}, u_{3}, u_{2}, v_{1}$ is a 5 cycle in $G$. However, this places us in Case 1 again, where $v_{1}$ and $v_{3}$ are playing the roles of $v_{1}$ and $v_{2}$, respectively.

This completes the proof.

We conclude this section by presenting a formula for $c(5, n)$ for all $n \geq 5$.

Theorem 8. For $n \geq 5, c(5, n)=2 n-1$.

Proof. Let $H=K(n-1, n-1)$ so that $\bar{H}=K_{n-1} \cup K_{n-1}$. The graph $H$ contains no 5-cycle, and $\bar{H}$ has no $n$-cycle; therefore, $c(5, n) \geq 2 n-1$. We verify that $c(5, n)=2 n-1$ by induction on $n(\geq 5)$, the result following for $n=5$ by Theorem 7.

Assume $c(5, n)=2 n-1$ for some $n \geq 5$, and let $G$ be a graph of order $2 n+1$. Since $c(5, n+1) \geq 2 n+1$, it suffices to show that $G$ contains a 5 -cycle or $\bar{G}$ contains an $(n+1)$-cycle. Assume that $G$ has no 5-cycle. Since $c(5, n)=2 n-1$, the graph $\bar{G}$ contains an $n$-cycle $C: u_{1}, u_{2}, \cdots, u_{n}, u_{1}$. Designate the remaining vertices by $v_{1}, v_{2}, \cdots, v_{n}$, and $v_{n+1}$.

If some $v_{i}(1 \leq i \leq n+1)$ is adjacent in $\bar{G}$ to two consecutive vertices of $C$, then $\bar{G}$ contains an $(n+1)$-cycle, completing the proof. Assume, therefore, that no $v_{i}$ is adjacent in $\bar{G}$ to two consecutive vertices of $C$. This implies that each $v_{i}$ is adjacent in $G$ to some set of $\{n / 2\}$ vertices of $C$, where at least one vertex in any pair of consecutive vertices of $C$ belongs to the set. If every two distinct $v_{i}$ are adjacent in $\bar{G}$, then $\bar{G}$ contains $K_{n+1}$ and hence $C_{n+1}$ as a subgraph. Suppose, then, that there are two distinct $v_{i}$, say $v_{1}$ and $v_{2}$, which are adjacent in $G$. We now consider three cases, assuming throughout that $v_{1} v_{2} \in E(G)$.

Case 1. Assume there is a vertex $v_{k}(k \neq 1,2)$ such that $v_{1}$ and $v_{k}$ are joined in $G$ to a vertex $u_{i}$ on $C$, and $v_{2}$ and $v_{k}$ are joined in $G$ to a vertex $u_{j}$ on $C$. If it is possible to select $u_{i}$ and $u_{j}$ such that $u_{i} \neq u_{j}$, then $G$ contains the 5-cycle $v_{k}, u_{i}, v_{1}, v_{2}, u_{j}, v_{k}$ which is contradictory. Hence we may suppose that $v_{1}$ and $v_{k}$ are joined in $G$ to only one vertex $u_{i}$ on $C$, and $v_{2}$ and $v_{k}$ are joined on $G$ to only one vertex on $C$, namely $u_{i}$. Since at least one vertex in every pair of consecutive vertices of $C$ is joined in $G$ to $v_{1}$ (respectively $v_{2}$ ), it follows that every vertex of $C$ different from $u_{i}$ is joined in $G$ to exactly one of $v_{1}$ and $v_{2}$. The vertex $v_{k}$ is adjacent in $G$ to at least $\{n / 2\}$ vertices of $C$; therefore, $v_{k}$ must be adjacent in $G$ to a vertex $u_{r}$ which is joined in $G$ to $v_{1}$, and, furthermore, $v_{k}$ is adjacent in $G$ to a vertex $u_{s}$ (different from $u_{r}$ ) which is joined in $G$ to $v_{2}$. Hence $G$ contains a 5-cycle, which is contrary to hypothesis.

We note that if $n$ is odd, Case 1 necessarily applies. We may henceforth assume $n$ to be even.

Case 2. Assume Case 1 does not bold and there exists some vertex $v_{k}(k \neq$ $1,2)$ which is adjacent in $G$ to no vertex of $C$ which is joined in $G$ to $v_{1}$ or $v_{2}$. 
This implies that whenever $v_{1} u_{i} \in E(G), 1 \leq i \leq n$, then $v_{k} u_{i} \in E(\bar{G})$, and whenever $v_{2} u_{j} \in E(G), 1 \leq j \leq n$, then $v_{k} u_{j} \in E(\bar{G})$. Since $v_{k}$ is joined in $G$ to at least $\{n / 2\}$ vertices of $C$, and $v_{k}$ is joined in $\bar{G}$ to at least $\{n / 2\}$ vertices of $C$, it follows that $v_{k}$ is adjacent in $G$ to exactly $n / 2$ vertices of $C$ and is adjacent in $\bar{G}$ to exactly $n / 2$ vertices of $C$. Therefore, we may assume here that $v_{1}$ and $v_{2}$ are joined in $G$ to the vertices of $S_{1}=\left\{u_{i} \mid i\right.$ is odd $\}$ and joined in $\bar{G}$ to the vertices of $S_{2}=\left\{u_{i} \mid i\right.$ is even $\}$, while $v_{k}$ is joined in $G$ to the vertices of $S_{2}$ and joined in $\bar{G}$ to the elements of $S_{1}$.

If $i$ and $j$ are both even, then $u_{i} u_{j} \in E(\bar{G})$; for otherwise, we may select an even $t \neq i, j$ (since $n \geq 6$ here) to obtain the 5-cycle $u_{i}, u_{j}, v_{2}, u_{t}, v_{1}, u_{i}$ of $G$, which is a contradiction. Then $\bar{G}$ contains the $(n+1)$-cycle $v_{k}, u_{n-1}, u_{n-2}, \cdots$, $u_{4}, u_{2}, v_{1}, u_{n}, u_{1}, v_{k}$, which gives the desired result.

Case 3. Assume that Case 1 and Case 2 do not hold. Hence each $v_{k}, k \geq 3$, has the properties that whenever $v_{1} u_{i}, v_{k} u_{i} \in E(G), 1 \leq i \leq n$, then $v_{2} u_{i} \in E(\bar{G})$, and whenever $v_{2} u_{j}, v_{k} u_{j} \in E(G), 1 \leq j \leq n$, then $v_{1} u_{j} \in E(\bar{G})$. Let $S_{1}$ and $S_{2}$ be defined as in Case 2. We may assume in this case that $v_{1}$ and $v_{3}$, say, are joined in $G$ to the elements of $S_{1}$ and joined in $\bar{G}$ to the elements of $S_{2}$, while $v_{2}$ is joined in $G$ to the vertices of $S_{2}$ and joined in $\bar{G}$ to the vertices of $S_{1}$.

If $v_{1} v_{3} \in E(G)$, then we have the conditions specified in Case 2, where $v_{1}$ and $v_{3}$ play the roles of $v_{1}$ and $v_{2}$, respectively. Hence, $v_{1} v_{3} \in E(\bar{G})$, and $\bar{G}$ contains the $(n+1)$-cycle $v_{1}, v_{3}, u_{2}, u_{3}, \cdots, u_{n}, v_{1}$.

The number $c(6,6)$. We next determine the value of $c(6,6)$.

Theorem 9. $c(6,6)=8$.

Proof. Let $H=K(2,5)$ so that $\bar{H}=K_{2} \cup K_{5}$. Since neither $H$ nor $\bar{H}$ has a 6 cycle, $c(6,6) \geq 8$. Let $G$ be a graph of order 8 , and suppose neither $G$ nor $\bar{G}$ contains a 6-cycle. We distinguish two cases.

Case 1. Assume neither $G$ nor $\bar{G}$ bas a 5-cycle. Since $c(4,4)=6$ by Theorem 3 , at least one of $G$ and $\bar{G}$ has a 4 -cycle; say $G$ contains the 4-cycle $C: u_{1}, u_{2}$, $u_{3}, u_{4}, u_{1}$. Denote the remaining vertices of $G$ by $v_{1}, v_{2}, v_{3}$, and $v_{4}$. Since $G$ has no 5-cycle, no $v_{i}$ is joined in $G$ to two consecutive vertices of $C$. This implies that every $v_{i}$ is joined in $\bar{G}$ to some pair of opposite vertices of $C$. We consider two subcases.

Subcase 1a. Suppose three or more $v_{i}$ are joined in $\bar{G}$ to the same pair of opposite vertices of $C$; say $v_{1}, v_{2}$, and $v_{3}$ are joined in $\bar{G}$ to $u_{1}$ and $u_{3}$. Every two distinct vertices in $\left\{v_{1}, v_{2}, v_{3}\right\}$ are adjacent in $G$, for otherwise $\bar{G}$ contains a 5cycle. Also, the vertex $v_{4}$ cannot be joined in $\bar{G}$ to two other $v_{i}$; otherwise, a 6 cycle exists in $\bar{G}$. Thus, we assume $v_{2} v_{4}$ and $v_{3} v_{4}$ are edges of $G$. Not both $u_{2} v_{2}$ and $u_{4} v_{3}$ are edges of $G$, for then $G$ contains a 6-cycle. Without loss of generality, we may assume $u_{2} v_{2}$ is an edge of $\bar{G}$. If $u_{2} v_{1}$ is an edge of $\bar{G}$, then $\bar{G}$ 
contains a 6-cycle. Furthermore, if $u_{2} v_{3} \in E(\bar{G})$, then $\bar{G}$ contains a 6-cycle. Therefore, $u_{2} v_{1}, u_{2} v_{3} \in E(G)$ and $G$ contains the 5-cycle $u_{2}, v_{1}, v_{2}, v_{4}, v_{3}, u_{2}$. This produces a contradiction.

Subcase 1b. Suppose exactly two $v_{i}$ are joined in $\bar{G}$ to the same pair of opposite vertices of $C$; say $v_{1}$ and $v_{2}$ are joined in $\bar{G}$ to $u_{2}$ and $u_{4}$ while $v_{3}$ and $v_{4}$ are joined in $\bar{G}$ to $u_{1}$ and $u_{3}$. Assume further that $v_{1} v_{3}$ is an edge of $\bar{G}$. The edge $v_{3} u_{2}$ belongs to $G$, for otherwise $v_{3}, u_{2}, v_{2}, u_{4}, v_{1}, v_{3}$ is a 5-cycle in $\bar{G}$. Similarly, 5-cycles result in $\bar{G}$ unless $v_{4} u_{2}$ and $v_{4} u_{4}$ are edges of $G$. Next, $v_{4} v_{3} \in E(\bar{G})$, or else $v_{4}, v_{3}, u_{2}, u_{3}, u_{4}, v_{4}$ is a 5-cycle in $G$. In a like manner, it follows that $v_{2} u_{1} \in E(G)$ and $v_{2} u_{3} \in E(\bar{G})$. However, $v_{2}, u_{3}, v_{4}, v_{3}, v_{1}, u_{4}, v_{2}$ is a 6 -cycle of $\bar{G}$ which is contradictory. Hence, we must have $v_{1} v_{3} \in E(G)$. By symmetry, we may also conclude that $v_{2} v_{3}, v_{1} v_{4}, v_{2} v_{4} \in E(G)$.

We observe that not both $u_{1} v_{2}$ and $u_{3} v_{1}$ are edges of $G$, for otherwise $u_{1}, v_{2}$, $v_{4}, v_{1}, u_{3}, u_{4}, u_{1}$ is a 6 -cycle of $G$. However, not both $u_{1} v_{2}$ and $u_{3} v_{1}$ are edges of $\bar{G}$ either, since then $u_{1}, v_{2}, u_{4}, v_{1}, u_{3}, v_{4}, u_{1}$ is a 6-cycle of $\bar{G}$. Thus, we may assume that $u_{1} v_{2} \in E(G)$ and $u_{3} v_{1} \in E(\bar{G})$. If the edge $u_{4} v_{4}$ is in $G$, then $G$ contains the contradictory G-cycle $u_{4}, v_{4}, v_{1}, v_{3}, v_{2}, u_{1}, u_{4}$. On the other hand, if $u_{4} v_{4}$ is in $\bar{G}$, then $\bar{G}$ contains the 6-cycle $u_{4}, v_{4}, u_{3}, v_{1}, u_{2}, v_{2}, u_{4}$. We, there fore, have a contradiction in this subcase, also.

Case 2. Assume that at least one of $G$ and $\bar{G}$ contains a 5-cycle. Without loss of generality, we assume that $G$ has the 5-cycle $C: u_{1}, u_{2}, u_{3}, u_{4}, u_{5}, u_{1}$ with the remaining vertices denoted by $v_{1}, v_{2}$, and $v_{3}$. Since $G$ has no 6 -cycles, no $v_{i}(1 \leq i \leq 3)$ is adjacent in $G$ to two consecutive vertices of $C$. Thus, each $v_{i}$ is joined in $\bar{G}$ to three nonconsecutive vertices of $C$.

We now make use of the following fact: If $S_{1}, S_{2}, S_{3}$ are 3-element subsets of a S-element set, then there exist $i, j(i \neq j)$ such that $\left|S_{i} \cap S_{j}\right| \geq 2$. Hence, if $S_{i}(i=1,2,3)$ denotes the set of three nonconsecutive vertices of $C$ which are joined in $\bar{G}$ to $v_{i}$, then there exist two vertices $v_{i}$, say $v_{1}$ and $v_{2}$, which are mutually adjacent in $\bar{G}$ to at least two vertices of $C$. This suggests a breakdown in. to two subcases.

Subcase 2a. Assume $v_{1}$ and $v_{2}$ are joined in $\bar{G}$ to the same three vertices of $C$; say $v_{1}$ and $v_{2}$ are adjacent in $\bar{G}$ to $u_{1}, u_{3}$, and $u_{4}$. If $v_{3}$ is joined in $\bar{G}$ to any two of the vertices $u_{1}, u_{3}$, and $u_{4}$, then it follows directly that $\bar{G}$ contains a 6 o cycle, which is contrary to hypothesis. Thus, we may assume that $v_{3}$ is joined in $\bar{G}$ to exactly one of $u_{1}, u_{3}$, and $u_{4}$. If $v_{3} u_{1} \in E(\bar{G})$, then we must have at least one of the edges $v_{3} u_{3}$ and $v_{3} u_{4}$ in $\bar{G}$ also; therefore, without loss of generality, we assume that $v_{3} u_{4} \in E(\bar{G})$. This further implies that $v_{3} u_{2}, v_{3} u_{5} \in E(\bar{G})$ and $v_{3} u_{1}, v_{3} u_{3} \in E(G)$. The edge $v_{2} u_{5}$ belongs to $G$, for otherwise $v_{2}, u_{5}, v_{3}, u_{4}, v_{1}$, $u_{3}, v_{2}$ is a 6 cycle of $\bar{G}$. In a like manner, it follows that $v_{2} u_{2}, v_{1} u_{5}, v_{1} u_{2} \epsilon$ $E(G)$. However, then, $v_{2}, u_{2}, u_{3}, v_{3}, u_{1}, u_{5}, v_{2}$ is a 6 -cycle of $G$, which is impossible. 
Subcase 2b. No two $v_{i}$ are joined in $\bar{G}$ to the same three vertices of $C$, but $v_{1}$ and $v_{2}$ are joined in $\bar{G}$ to two common vertices of $C$. Assume $v_{1}$ is adjacent in $\bar{G}$ with each of the vertices $u_{1}, u_{3}$, and $u_{4}$; thus, $v_{2}$ is adjacent in $\bar{G}$ with exactly two of the three vertices $u_{1}, u_{3}$, and $u_{4}$. The vertex $v_{2}$ cannot be joined in $\bar{G}$ to $u_{3}$ and $u_{4}$, for then $v_{2}$ must be joined in $\bar{G}$ to $u_{1}$ as well. Hence, without loss of generality, we assume $v_{2}$ is joined in $\bar{G}$ to $u_{1}$ and $u_{4}$. Necessarily, then, $v_{2} u_{2} \in E(\bar{G})$. We now consider the location of the edges $v_{3} u_{1}$ and $v_{3} u_{4}$, observing that not both $v_{3} u_{1}$ and $v_{3} u_{4}$ are in $\bar{G}$ (for this is the situation discussed in Subcase $2 a)$.

(i) If $v_{3}$ is joined in $\bar{G}$ to neither $u_{1}$ nor $u_{4}$, then, of course, $v_{3}$ is adjacent in $\bar{G}$ to $u_{2}, u_{3}$, and $u_{5}$. However, $\bar{G}$ contains the 6-cycle $v_{1}, u_{3}, v_{3}, u_{2}, v_{2}, u_{4}$, $v_{1}$, which is impossible.

(ii) Suppose $v_{3}$ is joined in $\bar{G}$ to only one of $u_{1}$ and $u_{4}$. Without loss of generality, we assume that $v_{3} u_{1} \in E(\bar{G})$. Unless $v_{3} u_{3}, v_{3} u_{5} \in E(\bar{G})$, we are returned to previously treated cases. However, $v_{1}, u_{3}, v_{3}, u_{1}, v_{2}, u_{4}, v_{1}$ is now a 6acycle of $\bar{G}$ which is a contradiction.

We summarize the values established for $c(m, n)$ in the following table.

\begin{tabular}{|c|c|c|c|c|c|c|c|}
\hline$y$ & 3 & 4 & 5 & 6 & 7 & 8 & \\
\hline 3 & 6 & 7 & 9 & 11 & 13 & 15 & . . . \\
\hline 4 & 7 & 6 & 7 & 7 & 8 & 9 & . . \\
\hline 5 & 9 & 7 & 9 & 11 & 13 & 15 & . . • \\
\hline 6 & 11 & 7 & 11 & 8 & & & \\
\hline 7 & 13 & 8 & 13 & & & & \\
\hline 8 & 15 & 9 & 15 & & & & \\
\hline & 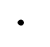 & • & • & & & & \\
\hline & $\cdot$ & . & • & & & & \\
\hline
\end{tabular}

\section{REFERENCES}

1. J. E. Graver and J. Yackel, Some graph theoretic results associated with Ramsey's theorem, J. Combinatorial Theory 4 (1968), 125-175. MR 37 \#1278.

2. F. Harary, Graph theory, Addison-Wesley, Reading, Mass., 1969. MR 41 \#1566.

3. F. P. Ramsey, On a problem of formal logic, Proc. London Math. Soc. 30 (1930), 264-286.

DEPARTMENT OF MATHEMATICS, WESTERN MICHIGAN UNIVERSITY, KALAMAZOO, MICHIGAN 49001

DE PARTMENT OF MATHEMATICS, CARLETON COLLEGE, NOR THFIELD, MINNESOTA 55057 